\title{
SIERRA LEONE RISE
}

\section{EN 066 39GGC \\ EN 066 21GGC \\ EN 066 32GGC}

Study of the dependence of core top ages for $G$ menardi and $G$ sacculifer on water depth in cores of low sedimentation rate $\left(\sim 2 \mathrm{~cm} / 10^{3} \mathrm{yr}\right)$. The study was initiated by Lisa Dubois of Brown University on cores originally studied by Curry and Lohmann of Woods Hole Oceanographic (see Table 2).

\section{REFERENCES}

Curry, W B and Lohmann, G P, 1983, Reduced advection into Atlantic Ocean deep eastern basins during last glaciation maximum: Nature, v 306, no. 5943, p 577-580.

_ 1986, Late Quaternary carbonate sedimentation at the Sierra Leone Rise (Eastern Equatorial Atlantic Ocean): Marine Geology, v 70, p 223-250.

TABLE 2

EN 066 39GGC Equatorial Atlantic Sierra Leone Rise Location $\left(5^{\circ} 04^{\prime} \mathrm{N}, 20^{\circ} 52^{\prime} \mathrm{W}\right)$ Depth $2818 \mathrm{~m}$

\begin{tabular}{|c|c|c|c|c|c|c|c|c|}
\hline $\begin{array}{l}\text { Depth } \\
(\mathrm{cm})\end{array}$ & $\begin{array}{c}\text { Coarse } \\
\text { fraction } \\
(\%)\end{array}$ & $\begin{array}{l}\text { Foram } \\
\quad \mathrm{Sp}\end{array}$ & $\begin{array}{l}\text { Abund } \\
\text { (no./gm) }\end{array}$ & $\begin{array}{l}\text { Abund } \\
\text { (mgm/gm) }\end{array}$ & $\begin{array}{c}\text { No. tests } \\
\text { Anal. }\end{array}$ & $\begin{array}{c}\text { Weight } \\
\text { Anal. } \\
\text { (mgm) }\end{array}$ & $\begin{array}{c}\text { Date of AMS } \\
\text { Analysis }\end{array}$ & $\begin{array}{l}\text { Age } \\
\text { (yr) }\end{array}$ \\
\hline $2-3$ & - & $\underline{G}$ menardi & - & - & - & 1.2 & Sept 85 & $1860 \pm 120$ \\
\hline " & - & $\bar{G} \overline{\operatorname{sacc}}$ & - & - & - & 12.1 & " & $4510 \pm 170$ \\
\hline$"$ & - & $\overline{\mathrm{M}} \overline{\text { benth }}$ & - & - & - & 9.0 & $"$ & $5180 \pm 180$ \\
\hline $10-11$ & - & $\underline{G}$ menardi & - & - & 100 & 10.3 & July 86 & $3920 \pm 90$ \\
\hline $11-12$ & - & $\underline{G}$ menardi & - & - & - & 10.1 & Sept 85 & $4100 \pm 160$ \\
\hline " & - & $\overline{\mathrm{G}} \overline{\mathrm{sacc}}$ & - & - & - & 8.0 & " & $7720 \pm 260$ \\
\hline " & - & $\overline{\mathrm{M}} \overline{\text { benth }}$ & - & - & - & 10.9 & $"$ & $10,430 \pm 350$ \\
\hline $13-14$ & - & $\underline{G}$ menardi & - & - & 66 & 3.0 & Aug 86 & $7510 \pm 200$ \\
\hline $16-17$ & - & $\underline{\mathrm{G}} \underline{\mathrm{sacc}}$ & - & - & 208 & 10.5 & Jan 87 & $15,130 \pm 280$ \\
\hline $23-24$ & - & $\underline{\mathrm{G}} \mathrm{sacc}$ & - & - & - & 11.6 & Sept 85 & $19,290 \pm 1080$ \\
\hline " & - & $\bar{G} \overline{i n f l a}$ & - & - & - & 5.5 & Feb 86 & $16,900 \pm 250$ \\
\hline$"$ & - & $\underline{\bar{M}} \overline{\text { benth }}$ & - & - & - & 5.7 & " & $20,430 \pm 360$ \\
\hline
\end{tabular}

EN 066 21GGC Equatorial Atlantic Sierra Leone Rise Location $\left(4^{\circ} 14^{\prime} \mathrm{N}, 20^{\circ} 38^{\prime} \mathrm{W}\right)$ Depth $3995 \mathrm{~m}$

\begin{tabular}{ccccccccc}
\hline $2-3$ & - & $\underline{G}$ menardi & - & - & - & 11.8 & Sept 85 & $2280 \pm 130$ \\
$"$ & - & $\underline{G}$ & - & - & - & 8.0 & $" ~$ & $3800 \pm 160$ \\
\hline
\end{tabular}

EN 066 32GGC Equatorial Atlantic Sierra Leone Rise Location $\left(2^{\circ} 28^{\prime} \mathrm{N}, 19^{\circ} 44^{\prime} \mathrm{W}\right)$ Depth $5003 \mathrm{~m}$

\begin{tabular}{|c|c|c|c|c|c|c|c|c|}
\hline $2-3$ & - & $\underline{G}$ menardi & - & - & - & 10.9 & Sept 85 & $2840 \pm 130$ \\
\hline$"$ & - & $\overline{\mathrm{G}} \overline{\mathrm{sacc}}$ & - & - & - & 8.1 & " & $4070 \pm 160$ \\
\hline
\end{tabular}

